\title{
Early Conversion to Aflibercept for Persistent Diabetic Macular Edema Results in Better Visual Outcomes and Lower Treatment Costs
}

This article was published in the following Dove Press journal: Clinical Ophthalmology

\author{
David J Ramsey ${ }^{1, *}$ \\ Samuel J Poulin ${ }^{1, *}$ \\ Lauren C LaMonica ${ }^{2}$ \\ Gregory R Blaha' \\ Fina C Barouch' \\ Jeffrey Chang' \\ Jeffrey L Marx' \\ 'Division of Ophthalmology, Lahey \\ Hospital \& Medical Center, Tufts \\ University School of Medicine, Peabody, \\ MA 01960, USA; ${ }^{2}$ University of Michigan \\ Medical School, Ann Arbor, MI 48109, \\ USA
}

*These authors contributed equally to this work
Purpose: To evaluate the functional and anatomic outcomes, as well as cost-effectiveness, of the timing of conversion to intravitreal aflibercept (IVA) in patients with treatmentresistant diabetic macular edema (DME).

Methods: Thirty consecutive eyes (25 patients) were identified that were treated with $\geq 3$ intravitreal bevacizumab (IVB) and/or ranibizumab (IVR) injections prior to treatment with $\geq 3$ IVA injections. Eyes that received $\leq 6$ IVB and/or IVR injections (earlyswitch) were compared to those that received $\geq 7$ injections (late-switch) prior to conversion to IVA. Treatment effectiveness was measured in quality-adjusted life years (QALYs). A micro-simulation model examined the impact of treatment duration on outcomes.

Results: Early- $(\mathrm{n}=18)$ and late- $(\mathrm{n}=12)$ switch eyes had similar vision prior to conversion to IVA. Despite improvements in retinal thickness, only the early-switch eyes maintained vision gains after conversion to IVA through the end of follow-up ( $p=0.027)$. Early switch saved \$22,884/eye and produced an additional 0.027 QALYs.

Conclusion: Early conversion to IVA optimizes vision outcomes and results in lower overall treatment expenditures.

Keywords: diabetic macular edema; DME, socioeconomics, diabetic retinopathy

\section{Introduction}

Swelling in the retina, known as diabetic macular edema (DME), is the leading cause of vision loss in working-age adults in the United States. ${ }^{1}$ Intravitreal injections of agents targeting the cytokine vascular endothelial growth factor (VEGF) have revolutionized treatment of DME, ${ }^{2-4}$ providing a greater chance of vision recovery compared to alternative treatments such as steroids or laser surgery. ${ }^{5}$ However, not all patients respond equally well to anti-VEGF medications, and some agents are costlier than others. A large clinical trial has demonstrated nearly equal efficacy of these medications as monotherapy for DME, ${ }^{6,7}$ but few studies have addressed the impact of changing agents when response to therapy is delayed or incomplete, ${ }^{8,9}$ or the cost-effectiveness of switching agents. ${ }^{10}$ The purpose of the present study is to evaluate the functional and anatomic outcomes, as well as cost-effectiveness, of the timing of conversion to intravitreal aflibercept (IVA) in patients with persistent DME previously treated with intravitreal bevacizumab (IVB) and/or ranibizumab (IVR).
Correspondence: David J Ramsey Lahey Hospital \& Medical Center, Tufts University School of Medicine, I Essex Center Drive, Peabody, MA 01960, USA Email David.J.Ramsey@lahey.org 


\section{Methods}

The study comprised a retrospective, cross-sectional comparative case series. The research followed the tenets of the Declaration of Helsinki and was approved by the institutional review board of Lahey Hospital \& Medical Center (Burlington, Massachusetts, USA). Using billing records, we identified patients who received $0.05 \mathrm{~mL}$ IVA injections for DME between January and June 2016. We excluded patients with retinal vein occlusions, ocular trauma, or treatment of choroidal neovascularization from other causes such as myopic degeneration, central serous retinopathy, or neovascular age-related macular degeneration. Criteria for inclusion included receipt of at least three IVA injections and at least three IVB or IVR injections prior to the switch to IVA. Patients who received laser or steroid treatment, or who had cataract extraction within three months of switching to IVA or within the IVA treatment period, were excluded. We also excluded individuals who received injections at other institutions or who had ocular or laser surgery within three months of the initiation of anti-VEGF treatment. Eyes that received six or fewer injections (early-switch) were compared to those that received seven or more injections (late-switch) prior to switching to IVA.

A retina sub-specialist reviewed the charts and extracted demographic and clinical data related to ocular health and diabetic retinopathy diagnosis, severity, and treatment. Hemoglobin A1c (A1c) values were obtained for all patients. Severity of diabetes was based on ICD$10-\mathrm{CM}$ codes. No patient had an increase in retinopathy severity level over the course of the study. The A1c value closest to the first and last injection date of each agent was used as an indicator of the severity of diabetes. In some cases, only one A1c value was collected, or the A1c measurement at the start of treatment was the last value obtained. Patients were assessed at each time point by Snellen visual acuity (VA), and central retinal thickness (CRT) was measured in microns by spectraldomain optical coherence tomography (OCT) scanning (Cirrus [Carl Zeiss Meditec, Inc] or Spectralis [Heidelberg Engineering, Inc, Heidelberg, Germany]). All measurements were converted to Heidelberg units for statistical analysis $(0.6 \times$ CRT[Cirrus] $+96.2 \mu \mathrm{m}) .{ }^{11}$

A reference group of eyes from patients who received IVB and/or IVR but who did not switch to IVA was developed. This group matched the early-switch group within 1.5 standard deviations of the mean total treatment duration and number of injections. The same exclusion criteria applied to this reference group. For additional comparison, a separate "reconfigured" switch group was constructed whereby late-switch patients were reconfigured into early-switch patients to assess their VA and CRT as if they had received IVA injections at an earlier time point instead of continuing on treatment with IVB and/or IVR. For this analysis, the date of late-switch patients' first IVA injection was reconfigured as the third injection pre-IVA, the second IVA injection as the second injection pre-IVA, the third injection as the first injection pre-IVA, and the fourth IVA injection as the first IVA injection.

The treatment index was defined as a ratio of the number of intravitreal injections a patient received during the treatment period compared with the maximum number of injections possible during that period based on product labeling. The maximum number of possible injections was determined using a maximum injection frequency of one injection every 28 days. The treatment burden was reported as a percentage, with higher values indicating a larger treatment burden, and a value of $100 \%$ indicating that a patient received one injection every 28 days during the entire treatment period.

A micro-simulation model examined the impact of the duration of treatment and time of switch to IVA on outcomes. Patient quality of life was assessed using QALYs, calculated at each visit by assigning a utility value to the patient's measured VA based upon conversion tables in Brown et al. ${ }^{12}$ In this study, VA in a patient's better-seeing eye was linked with health-related quality of life. Monthly utility values were summed to provide yearly QALYs gained per patient and projected over 5 and 10 years by using the patient's final VA measurement. QALYs were subsequently adjusted for duration of treatment at conversion to IVA and end of follow-up. ${ }^{13}$ After the first year, QALY values were discounted at $3.5 \%$ annually. ${ }^{14}$ Treatment costs were estimated for each treated eye by utilizing average Medicare reimbursement rates for the injection procedure (\$102.97) and adding the cost of one vial of each of the three anti-VEGF agents (IVA: \$1850; IVB: \$60; IVR: \$1170). ${ }^{13}$ Cost savings for two eyes treated during the same visit were disregarded for the purposes of this analysis. QALYs gained and the total cost of treatment were used to calculate the net monetary benefit (NMB) of treatment at a willingness-to-pay (WTP) threshold of $\$ 100,000 / \mathrm{QALY}$ using the formula NMB $=$ QALY gained x WTP - Cost. $^{13-16}$ 


\section{Statistical Analysis}

Data were analyzed using SPSS ${ }^{\circledR}$ Statistics version 22.0 (IBM Corp, Armonk, NY). Data are presented as mean $( \pm \mathrm{SD})$ for continuous variables. VA was transformed from presenting Snellen acuity to a log minimum angle of resolution (logMAR) scale. We used Student's $t$-test ( $t$-test), analysis of variance (ANOVA), and analysis of covariance (ANCOVA) to compare continuous variables and logistic regression to analyze categorical variables. Significance was judged at the $5 \%$ level $(p<0.05)$. KaplanMeier survival analysis was used to assess the time-toconversion to IVA.

\section{Results}

Two hundred sixty-eight eyes (212 patients) with DME that received anti-VEGF injections between 2010 and 2016 were identified. Thirty eyes that received at least three injections of IVB and/or IVR prior to switching to IVA in 25 patients were included in this case series. Thirteen (43\%) of these eyes were in female patients. Three eyes from three patients had Type 1 diabetes $(10 \%)$. The mean age of the patients was 64.9 years $( \pm 11.9$ years). Ninety-two percent of patients were Caucasian. During follow-up, no ocular or systemic side effects were observed that could be attributed to intravitreal injections. No eyes developed complications from neovascularization, nor did they develop new glaucoma during the course of the study. Fifty-three percent of eyes were pseudophakic at the time of switch to IVA. No patient had lens extraction within three months of switch to IVA or in the IVA treatment period. Additional characteristics of the study population are presented in Table 1 and Supplemental Table 1.

\section{Visual and Anatomical Outcomes}

Early- $(\mathrm{n}=18)$ and late- $(\mathrm{n}=12)$ switch eyes had similar vision and CRT at baseline, as well as prior to the conversion to IVA (Figure 1). In early-switch eyes, VA did not significantly improve from baseline to the time of the switch to IVA (logMAR $0.60 \pm 0.46$ to $0.60 \pm 0.32$, $p=0.490$ ). However, after the switch to IVA, a modest VA improvement was noted at the last follow-up ( $\log$ MAR $0.45 \pm 0.31, p=0.027)$. In late-switch eyes, there was no improvement in VA by the time of the switch ( $\log$ MAR $0.61 \pm 0.56$ to $0.51 \pm 0.31, p=0.442$ ), and there was no further gain in vision through the end of followup after the switch to IVA ( $\log$ MAR $0.52 \pm 0.31, p=0.236$ ).
Table I Demographic and Clinical Characteristics

\begin{tabular}{|c|c|c|c|}
\hline Age (Years) & \multicolumn{2}{|c|}{ Mean (SD) } & Significance \\
\hline All switch patients $(n=25)$ & \multicolumn{2}{|c|}{$64.9(11.9)$} & \\
\hline Early-switch $(n=15)$ & \multicolumn{2}{|c|}{$65.5(11.4)$} & $t_{p}=0.480$ \\
\hline Late-switch $(n=10)$ & \multicolumn{2}{|c|}{$62.0(13.0)$} & \\
\hline Reference $(n=19)$ & \multicolumn{2}{|c|}{$69.4(11.2)$} & $\S_{p}=0.192^{\S}$ \\
\hline Gender (\%) & Female & Male & Significance \\
\hline All switch patients & $44.0 \%$ & $56.0 \%$ & \\
\hline Early-switch & $46.7 \%$ & $53.3 \%$ & $t_{p}=0.742$ \\
\hline Late-switch & $40.0 \%$ & $60.0 \%$ & \\
\hline Reference & $22.2 \%$ & $79.9 \%$ & ${ }^{\S_{p}}=0.037$ \\
\hline Race (\%) & White & Other & Significance \\
\hline All switch patients & $92.0 \%$ & $8.0 \%$ & \\
\hline Early-switch & $94.4 \%$ & $5.6 \%$ & $t_{p}=0.763$ \\
\hline Late-switch & $91.7 \%$ & $8.3 \%$ & \\
\hline Reference & $79.0 \%$ & $21.0 \%$ & $\S_{p}=0.532$ \\
\hline Lens Status (\%) & \multicolumn{2}{|c|}{ Pseudophakic } & Significance \\
\hline All switch eyes $(n=30)$ & \multicolumn{2}{|l|}{$53.3 \%$} & \\
\hline Early-switch $(n=18)$ & \multicolumn{2}{|l|}{$55.5 \%$} & $t_{p}=0.765$ \\
\hline Late-switch $(n=12)$ & \multicolumn{2}{|l|}{$50.0 \%$} & \\
\hline Reference & \multicolumn{2}{|l|}{$68.4 \%$} & $\S_{p}=0.272$ \\
\hline
\end{tabular}

Notes: ${ }^{\dagger} p$-value calculated for the early- versus late-switch groups. ${ }^{\delta} p$-value calculated for the reference versus the all-switch groups.

The total improvement in vision over the entire treatment period was modest for all eyes $(\triangle \log M A R-0.13 \pm 42$, $p=0.044)$, with a trend toward more vision gained in eyes switched early $(\Delta \log M A R-0.16 \pm 0.41)$ compared with those that switched late $(\Delta \log M A R-0.097 \pm 0.45)$.

Both early- and late-switch groups demonstrated a near continuous improvement in CRT over the course of treatment, regardless of agent (Figure 1). In early-switch eyes, the CRT improved from $412 \pm 73 \mu \mathrm{m}$ at baseline to 382 $\pm 114 \mu \mathrm{m}$ at the time of the switch, and $306 \pm 81 \mu \mathrm{m}$ by the end of follow-up $(p<0.001)$. In late-switch eyes, the CRT improved from $465 \pm 140 \mu \mathrm{m}$ at baseline to $335 \pm 52 \mu \mathrm{m}$ at the time of the switch, and to $271 \pm 56 \mu \mathrm{m}$ by the end of follow-up $(p<0.001)$.

In comparison with early- and late-switch eyes, reference eyes gained vision by the equivalent point at which treatment-refractory eyes switched to IVA ( $\log$ MAR $-0.17 \pm 0.27$, $p=0.016$ ) and continued to gain vision through the end of follow-up $(\triangle \log M A R-0.24 \pm 0.28)$ compared with the treatment refractory group $(\Delta \log M A R-0.13 \pm 42, p=0.001)$. These reference eyes also demonstrated a steady improvement in macular anatomy, which may also explain why these patients were not switched to IVA (Figure 1). 

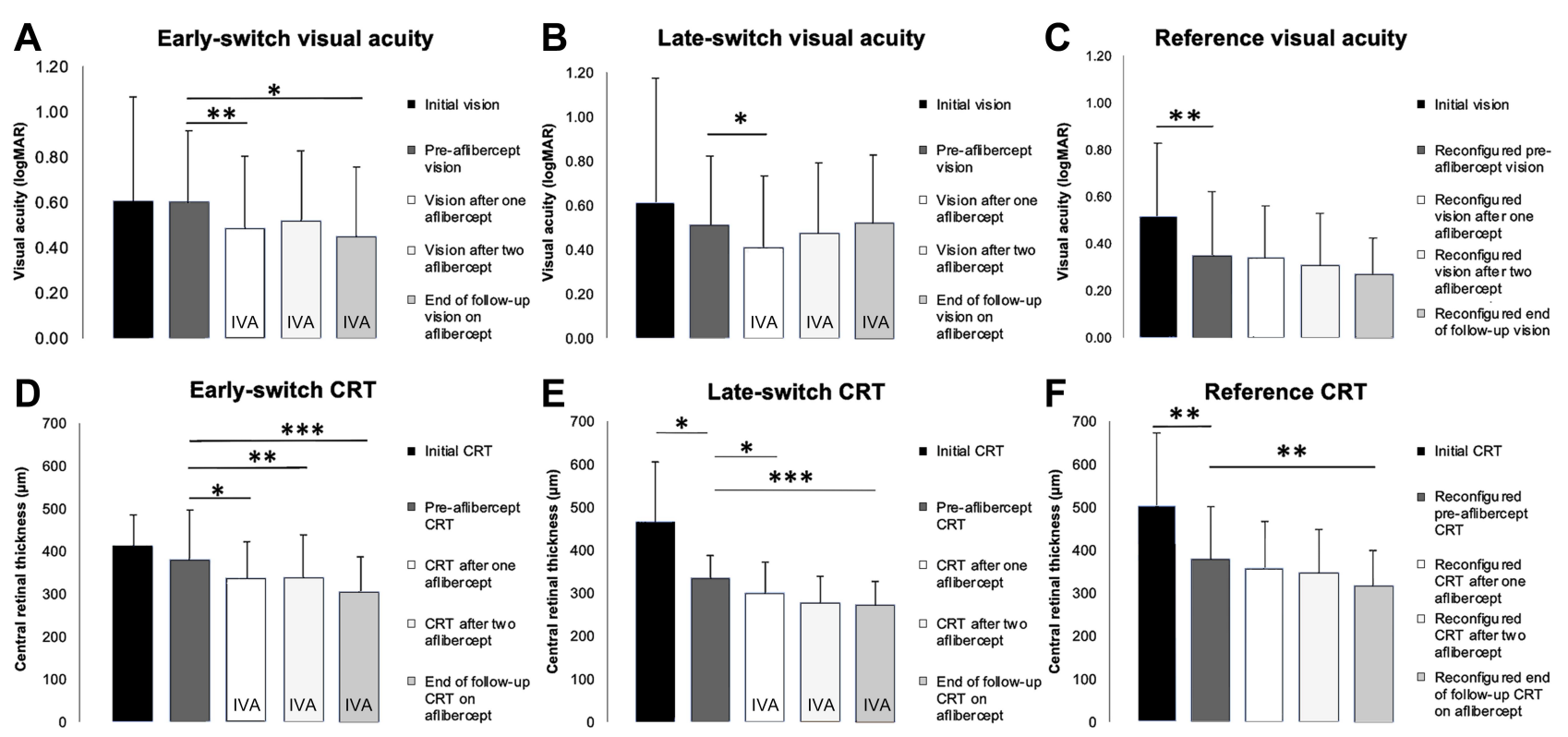

Figure I Visual acuity (VA) and central retinal thickness (CRT) outcomes of early- and late-switch patients converted to intravitreal aflibercept. VA before and after conversion to IVA in patients with DME who were (A) switched after six or fewer injections of IVR and/or IVB (early-switch, $n=18$ ), (B) switched after seven or more injections of IVR and/or IVB (late-switch, $n=12$ ), or (C) not switched to IVA (reference). Snellen VA was converted to logMAR units for this analysis. CRT before and after conversion to IVA in patients with DME who were (D) switched after 6 or fewer injections of IVR and/or IVB (early-switch), (E) switched after seven or more injections of IVR and/or IVB (late-switch), or $(\mathbf{F})$ not switched to IVA (reference, $\mathrm{n}=18$ ). Measurement periods were matched in the reference group for similar treatment duration and number of injections as the group converted early to IVA during treatment. Data are presented as the mean \pm standard error of mean (SEM). $p$-values were calculated using Student's t-test $(* p<0.05, * * p<0.01, * * * p<0.001)$

Late-switch eyes reconfigured to allow for comparison of VA and anatomical outcomes, as if they had received IVA injections at an earlier point, had no statistically significant VA difference in comparison with early-switch eyes at all time points. Prior to the switch to IVA, reconfigured late-switch eyes had similar vision to early-switch eyes (logMAR $0.60 \pm 0.17$ versus $0.61 \pm 0.08, p=0.958)$. Similarly, reconfigured late-switch eyes had indistinguishable CRT compared with early-switch eyes prior to the switch to IVA ( $\log$ MAR $430 \pm 38 \mu \mathrm{m}$ versus $377 \pm 25 \mu \mathrm{m}, \quad p=0.262$ ). These reconfigured eyes did not demonstrate any shortterm improvement in vision following the simulated switch to IVA ( $\log$ MAR $0.52 \pm 0.53$ versus $0.60 \pm 0.54$, $\mathrm{p}=0.732$ ), as was the case for our study eyes following the actual conversion to IVA, nor did they experience any visual gains from switch to IVA through end of follow-up when measured from this reconfigured time point ( $\log$ MAR $0.52 \pm 0.53$ versus $0.52 \pm 0.31, \mathrm{p}=0.970$ ).

\section{Treatment Index}

Early-switch eyes received an average of $4.56 \pm 1.20$ injections over a median period of 5.9 months prior to switching to IVA, whereas late-switch eyes received an average of $23.0 \pm 14.6$ injections over a median period of 24 months prior to switching to IVA $(p<0.001)$. Early-switch eyes received $74.2 \%$ of injections possible within the preswitch period, compared with $80.0 \%$ of injections possible for late-switch eyes $(p=0.280)$. Early-switch eyes received injections an average of every 5.6 \pm 1.3 weeks, compared with late-switch eyes that received injections an average of $5.1 \pm 0.8$ weeks $(p=0.191)$. Time-to-conversion to IVA is presented in Supplemental Figure 1.

After conversion to IVA, early-switch eyes received an average of $6.44 \pm 4.82$ injections over a median period of 6.7 months, and late-switch eyes received an average of $6.41 \pm 3.70$ injections over a median period of 7.3 months $(p=0.987)$. No difference in treatment index was noted after the switch for early-switch compared with lateswitch eyes $(71.9 \%$ versus $69.1 \%, p=0.624)$. After the switch to IVA, both groups had slightly longer injection intervals. Early-switch eyes received IVA injections an average of every $6.4 \pm 1.9$ weeks compared with lateswitch eyes that received IVA injections an average of every $6.5 \pm 1.7$ weeks $(p=0.898)$.

Reference eyes received an average of $9.84 \pm 1.26$ injections over a median period of 12.8 months. The number of injections was similar to the early-switch eyes by design. Additional treatment characteristics of the study population are presented in Supplemental Table 2. 


\section{Factors Related to the Switch to Aflibercept}

Several variables demonstrated a correlation with the number of injections that a patient received prior to the switch to aflibercept. The change in CRT by the point of the switch $\left(\mathrm{R}^{2}=0.425, p<0.001\right)$ had the strongest correlation with the number of injections a patient received by the time of the switch to IVA. By comparison, CRT at the start of treatment $\left(\mathrm{R}^{2}=0.353, \mathrm{p}<0.001\right)$ or at the switch $\left(\mathrm{R}^{2}=0.134, p=0.046\right)$ was not as strongly correlated with number of injections. Although VA at the start of treatment or at the visit of the switch to IVA did not correlate with the total number of injections up to the point of the switch, the change in vision from the start of treatment to the point at which treatment was switched did have a modest correlation $\left(\mathrm{R}^{2}=0.220\right.$, $p=0.009$ ), with a greater change in vision associated with a later switch to IVA. However, final visual outcome was not correlated with either the total number of injections, number of pre-switch injections, or duration of treatment.

\section{Cost-Effectiveness of Treatment}

A microsimulation analysis was conducted on the study population to identify cost of treatment for early- and lateswitch patients, as well as for a reference group matched for similar treatment duration and number of injections as the early-switch group. Early-switch patients saved \$22,885 per eye over the course of treatment compared to late-switch patients, while producing an additional 0.027 QALYs/eye, which yields an NMB $>\$ 25,000$ /eye (Figure 2). Incremental

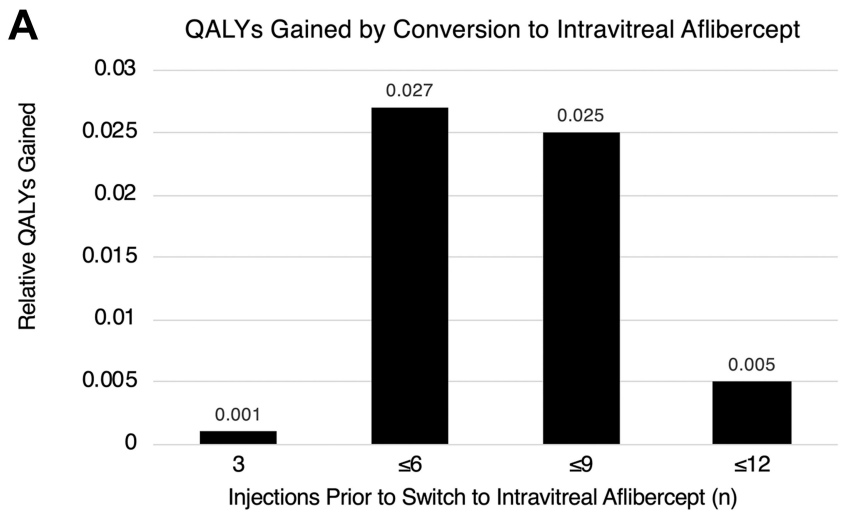

QALYs gained by early switch and projected at 5- and 10years are presented in Table 2. Relative QALYs gained reached a maximum with six or fewer pre-switch injections (Figure 2A), whereas cost savings and NMB accrued the longer that the switch to IVA was delayed up to 12 preswitch injections (Figure 2B). Modeling the cost savings as if all patients had been treated with IVB before the switch to IVA would decrease the savings per eye to $\$ 2,843$. Early switch to IVA was not less costly at any point in time compared with the reference group (Table 3). Total cost was associated with baseline $\mathrm{CRT}\left(\mathrm{R}^{2}=0.280, p=0.003\right)$, but not with VA, age, gender, type or severity of diabetes.

\section{Discussion}

DME is the most common vision-threatening complication of diabetic retinopathy and is a condition of increasing socioeconomic concern owing to the growing US population with diabetes. ${ }^{1,10}$ The efficacy and the ideal timing for switching to IVA for treatment-refractory DME have remained undetermined to date. ${ }^{9}$ Our study found that early conversion to IVA for persistent DME appears to maximize the benefit of the treatment while reducing both the duration and cost of treatment. Despite sustained improvement in retinal anatomy in both early- and lateswitch groups, only patients who were switched early to IVA maintained VA gains through the end of follow-up. Delaying switch in treatment for refractory DME may contribute to suboptimal outcomes during the period over which DME is inadequately managed, which could result in irreversible structural damage to the foveal center.

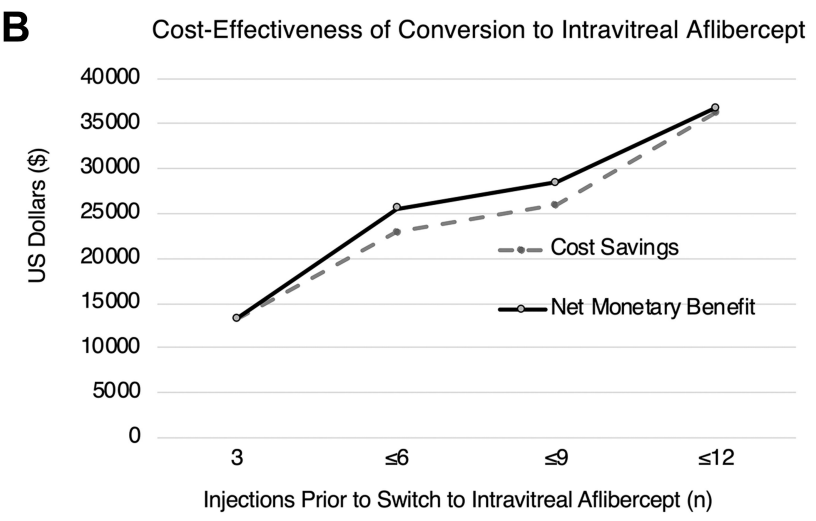

Figure 2 Early switch to intravitreal aflibercept is most cost-effective at six or fewer pre-switch injections. (A) QALYs gained by conversion to intravitreal aflibercept at various number of injections prior to switch. VA in logMAR units was converted directly into QALY units for analysis. Bars represent mean $\mathrm{QALYs}$ gained. $\mathrm{N}=30$ eyes. (B) Cost-effectiveness of conversion to intravitreal aflibercept at various number of injections prior to switch. Treatment costs were estimated for each treated eye by utilizing average Medicare reimbursement rates for injection procedure and agent. ${ }^{13} \mathrm{~A}$ microsimulation model was generated to calculate cost savings over the course of treatment (dashed line), which is the difference in total treatment cost based on treatment group, as well as the net monetary benefit (solid line) accounting for additional QALYs gained at a WTP threshold of $\$ 100,000 / Q A L Y$. QALYs and cost savings were realized even after controlling for the number of post-switch injections in early- (6.4 \pm 4.5$)$ and late- $(6.4 \pm 3.7)$ switch eyes $(p=0.987)$. The number of post-switch injections for our patient population was comparable to other studies investigating the impact of IVA on clinical outcomes. ${ }^{3}$ 
Table 2 Quality-Adjusted Life Years (QALYs), Adjusted and Predicted

\begin{tabular}{|c|c|c|c|c|c|c|}
\hline & \multicolumn{6}{|c|}{ Average QALYs, Adjusted } \\
\hline & \multicolumn{2}{|c|}{$\begin{array}{l}\text { Start of } \\
\text { Treatment }\end{array}$} & \multicolumn{2}{|c|}{$\begin{array}{l}\text { Conversion } \\
\text { to IVA }\end{array}$} & \multicolumn{2}{|c|}{$\begin{array}{l}\text { End of } \\
\text { Follow-Up }\end{array}$} \\
\hline & Mean & \pm SD & Mean & \pm SD & Mean & $\mathbf{\pm S D}$ \\
\hline Early switch & 0.74 & 0.08 & 0.74 & 0.08 & 0.77 & 0.08 \\
\hline Late switch & 0.74 & 0.13 & 0.73 & 0.08 & 0.71 & 0.08 \\
\hline \multirow[t]{4}{*}{ Reference } & 0.75 & 0.08 & 0.80 & 0.07 & 0.81 & 0.06 \\
\hline & \multicolumn{6}{|c|}{ Average QALYs, Predicted } \\
\hline & \multicolumn{2}{|c|}{$\begin{array}{l}\text { I-Year } \\
\text { Horizon }\end{array}$} & \multicolumn{2}{|c|}{$\begin{array}{l}\text { 5-Year } \\
\text { Horizon }\end{array}$} & \multicolumn{2}{|c|}{$\begin{array}{l}\text { 10-Year } \\
\text { Horizon }\end{array}$} \\
\hline & Mean & \pm SD & Mean & \pm SD & Mean & $\pm \mathbf{S D}$ \\
\hline Early switch & 0.74 & 0.07 & 3.56 & 0.33 & 6.60 & 0.65 \\
\hline Late switch & 0.75 & 0.11 & 3.55 & 0.37 & 6.52 & 0.61 \\
\hline Reference & 0.79 & 0.05 & 3.79 & 0.23 & 6.99 & 0.45 \\
\hline
\end{tabular}

Alternatively, differences in VA may be due to the presence of iatrogenic vision loss from prior treatments, such as from the expansion of laser scars, progressive macular ischemia, or due to the loss of retinal function from longterm growth factor suppression related to anti-VEGF agents themselves, as has been observed in macular degeneration. ${ }^{17}$

Recent meta-analyses of nonrandomized studies of patients switched from IVB and/or IVR to IVA for treatment-resistant DME suggest that IVA may provide superior visual and anatomical outcomes in comparison to IVR and/or IVB. ${ }^{10,18}$ The weighted mean gain in bestcorrected VA (logMAR 0.09 , CI 0.03 to 0.14 ) and CRT $(100.55 \mu \mathrm{m}, \mathrm{CI} 68.46 \text { to } 132.63)^{10}$ from these studies is

Table 3 Early Conversion to Intravitreal Aflibercept is More Cost-Effective Than Late Conversion

\begin{tabular}{|l|l|l|l|}
\hline & $\begin{array}{l}\text { Total } \\
\text { Treatment }\end{array}$ & $\begin{array}{l}\text { Start of } \\
\text { Treatment } \\
\text { to Conversion } \\
\text { to IVA }\end{array}$ & $\begin{array}{l}\text { Conversion to } \\
\text { IVA to End of } \\
\text { Follow-Up }\end{array}$ \\
\cline { 2 - 4 } & All Patients & All Patients & All Patients \\
\hline $\begin{array}{l}\text { Early Switch } \\
\text { Late Switch } \\
\text { Reference }\end{array}$ & $\$ 15,040$ & $\$ 2563$ & $\$ 12,477$ \\
\hline $\begin{array}{l}\$ 37,925 \\
\text { Cost Savings of Early } \\
\text { Conversion }\end{array}$ & $\$ 5460$ & $\$ 2055$ & $\begin{array}{l}\$ 2,369 \\
\$ 3363\end{array}$ \\
\hline
\end{tabular}

remarkably similar to the total VA gain (logMAR 0.09 $\pm 0.05)$ and CRT reduction $(71.6 \pm 10.5 \mu \mathrm{m})$ observed through end of follow-up in eyes switched early to IVA in our study. Although these gains are quite modest, some eyes experience more substantial improvements than others. Other studies have found no sustained improvement in VA after the switch to IVA, but these studies did not differentiate patients based on timing of the switch. ${ }^{8}$ Some studies have even excluded eyes switched after longer durations of anti-VEGF treatment, ${ }^{9}$ which are similar to the late-switch eyes in our study that experienced more modest effects on vision and retinal thickness.

In clinical practice, both physicians and patients face difficult decisions in regard to individualizing treatment. They may seek a change in therapy even when there is insufficient evidence of a primary treatment failure. ${ }^{19}$ A desire to do what is best for a patient may not always be influenced by taking cost into consideration, since neither the patient nor physician often directly bears this burden of treatment. Clinical experience and a number of case series indicate that it may be worthwhile to switch to another anti-VEGF agent when there is evidence for limited treatment efficacy. ${ }^{8-10,18,20,21}$ However, clinical trials have shown that patients with DME often require additional injections over the course of months, or even years, after primary endpoints are reached. ${ }^{7,22}$ Studies have also shown that by simply continuing anti-VEGF treatment, some patients with a limited anatomic response to treatment will experience VA gains and improvement in CRT similar to those patients identified as having an immediate response. ${ }^{23,24}$ A sub-analysis of the Diabetic Retinopathy Clinical Research Network Protocol I study showed that patients with persistent DME during the first six months of treatment still gained significant vision through three years with continuous treatment. ${ }^{25}$ Ferris et al. ${ }^{19}$ evaluated eyes that met several defined criteria for "switching" treatment but nonetheless continued on their originally assigned treatment in the Diabetic Retinopathy Clinical Research Network studies. Analysis of these eyes found a mean VA improvement of three- to five-letters and CRT reduction of $40-70 \mu \mathrm{m}$ three months beyond the point at which treatment first appeared to be ineffective. This finding illustrates the challenges of differentiating effects due to a switch in treatment from those due to regression to the mean or to time effects related to continued treatment without a true comparison group. ${ }^{19}$ 
To date, no study for refractory DME has utilized a comparison group. ${ }^{8-10,18}$ We compare the eyes switched to IVA in our study to a reference group of eyes that did not undergo a switch. We also reconfigure our late-switch eyes to show that a late switch was not associated with tachyphylaxis after earlier treatment success. However, the long-term outcomes of sequential or combination treatment with anti-VEGF agents (or steroid treatment) remain unclear, and no randomized controlled trials have systematically evaluated whether switching between anti-VEGF agents may be more effective for DME.

Our results highlight the difficult decisions that physicians, patients, and policymakers face when the costs of treatment and efficacy of results are at odds. Many factors must be considered when deciding whether to switch a patient from one agent to another, including access to therapy, availability of drug, adherence to treatment criteria, application of treatment failure and re-treatment criteria, as well as the impact of treatment burden on patients. IVA is a more effective drying agent ${ }^{2}$ - having a duration of VEGF suppression twice as long compared with IVR; ${ }^{26}$ its potential to aid in treatment based on anatomical, if not visual outcomes, may be a factor driving the decision to switch some patients from other agents. ${ }^{8}$

The limitations of the present study include its retrospective nature, small sample size, and variations in the number and frequency of injections, typical of real-world treatment of patients with DME. The population in our study is derived from a single center, outpatient clinic that serves as a retina referral center, which may select for more severe cases of diabetic retinopathy or treatment-resistant DME. Presenting VA is also not equivalent to best-corrected VA or ETDRS vision used in randomized clinical trials, as Snellen acuity has been shown to underestimate VA. ${ }^{27}$ This limits our ability to compare our findings with clinical trials, and it may also underestimate the impact of the switch to IVA on vision. Lastly, although we controlled for clinical characteristics and events that may be expected to affect vision and anatomical outcomes, our microsimulation and regression analyses do not control for insurance approval of the three anti-VEGF agents, sociodemographic characteristics, or other non-clinical factors that may affect a patient's ability to switch agents.

\section{Conclusion}

Our microsimulation model demonstrates that switching early to IVA by six or fewer anti-VEGF injections optimizes visual outcomes and results in lower overall treatment expenditures compared with later switch at all time points. Identifying patients with delayed treatment response to IVR and/or IVB and switching patients early to IVA may not only reduce vision loss, but also lower total treatment costs. These findings reveal a significant area of opportunity not only to increase patient quality of life, but also to reduce the cost burden associated with anti-VEGF treatment to our health care system.

\section{Abbreviations}

DME, diabetic macular edema; IVA, intravitreal aflibercept; IVB, intravitreal bevacizumab; IVR, intravitreal ranibizumab; CRT, central retinal thickness; QALYs, quality-adjusted life years; VEGF, vascular endothelial growth factor; VA, visual acuity; OCT, optical coherence tomography; logMAR, log minimum angle of resolution; NMB, net monetary benefit; WTP, willingness-to-pay; ICD-10-CM, International Classification of Diseases, 10th Revision, Clinical Modification.

\section{Ethical Approval}

This case series was conducted in accordance with the Declaration of Helsinki. The collection and evaluation of all protected patient health information was performed in a HIPAA (Health Insurance Portability and Accountability Act)-compliant manner. This study was approved by the institutional review board of the Lahey Hospital \& Medical Center (Burlington, Massachusetts, USA): Evaluation of treatment response to anti-VEGF injections [765,268-13].

\section{Statement of Informed Consent}

This study received institutional review board approval. A waiver of informed consent was obtained for the research and publication of this article.

\section{Acknowledgments}

The authors thank Dr. David Kim, Dr. David Lazar, Dr. Noreen Shaikh, Dr. Shiyoung Roh, Christine Gould, Miranda Prosniewski, and Carol Spencer, Lahey Hospital Librarian, for research support. D.J. Ramsey is the Harry N. Lee Family Chair in Innovation at the Lahey Hospital \& Medical Center, Beth Israel Lahey Health. This article was presented at the The American Academy of Ophthalmology, Chicago, IL, October 29, 2018 with interim findings. The poster's abstract was published in 'Poster Abstracts' in the meeting program (PO480). D. J. Ramsey and S.J. Poulin are co-first authors. 


\section{Funding}

S.P.: Supported by a grant from Office of Medical Education, Lahey Hospital \& Medical Center, Beth Israel Lahey Health. D.J.R.: Supported by the Harry N. Lee Family Chair in Innovation at the Lahey Hospital \& Medical Center, Beth Israel Lahey Health.

\section{Disclosure}

The authors declared no potential conflicts of interest with respect to the research, authorship, publication of this article, and this work.

\section{References}

1. Cheung N, Mitchell P, Wong TY. Diabetic retinopathy. Lancet. 2010;376(9735):124-136. doi:10.1016/S0140-6736(09)62124-3

2. Diabetic Retinopathy Clinical Research Network, Wells JA, Glassman AR, et al. Aflibercept, bevacizumab, or ranibizumab for diabetic macular edema. $N$ Engl J Med. 2015;372(13):1193-1203.

3. Nguyen QD, Brown DM, Marcus DM, et al. Ranibizumab for diabetic macular edema: results from 2 Phase III randomized trials: RISE and RIDE. Ophthalmology. 2012;119(4):789-801. doi:10.1016/j.ophtha.2011.12.039

4. Korobelnick JF, Do DV, Schmidt-Erfuth U, et al. Intravitreal aflibercept for diabetic macular edema. Ophthalmology. 2014;121 (11):2247-2254. doi:10.1016/j.ophtha.2014.05.006

5. Diabetic Retinopathy Clinical Research Network, Elman MJ, Aiello LP, et al. Randomized trial evaluating ranibizumab plus prompt or deferred laser or triamcinolone plus prompt laser for diabetic macular edema. Ophthalmology. 2010;117(6):1064-1077 e35. doi:10.1016/j.ophtha.2010.02.031.

6. Wells JA, Glassman AR, Ayala AR, et al. Aflibercept, bevacizumab, or ranibizumab for diabetic macular edema: two-year results from a comparative effectiveness randomized clinical trial. Ophthalmology. 2016;123(6):1351-1359. doi:10.1016/j.ophtha.2016.02.022

7. Brown DM, Nguyen QD, Marcus DM, et al. Long-term outcomes of ranibizumab therapy for diabetic macular edema: the 36-month results from two phase III trials: RISE and RIDE. Ophthalmology. 2013;120(10):2013-2022. doi:10.1016/j.ophtha.2013.02.034

8. Rahimy E, Shahlaee A, Khan MA, et al. Conversion to aflibercept after prior anti-VEGF therapy for persistent diabetic macular edema. $\mathrm{Am}$ J Ophthalmol. 2016;164:118-127.e2. doi:10.1016/j.ajo.2015.12.030

9. Ashraf M, Souka AA, ElKayal H. Short-term effects of early switching to ranibizumab or aflibercept in diabetic macular edema cases with non-response to bevacizumab. Ophthalmic Surg Lasers Imaging Retina. 2017;48(3):230-236. doi:10.3928/23258160-20170301-06

10. Xiao K, Li FZ, Liang SZ, Wang J, Qian C, Wan GM. Efficacy of conversion to aflibercept for diabetic macular edema previously refractory to bevacizumab or ranibizumab: a meta-analysis of high-quality nonrandomized studies. Ann Pharmacother. 2020;54 (8):750-756. doi:10.1177/1060028020904358

11. Giani A, Cigada M, Choudhry N, et al. Reproducibility of retinal thickness measurements on normal and pathologic eyes by different optical coherence tomography instruments. Am J Ophthalmol. 2010;150(6):815-824. doi:10.1016/j.ajo.2010.06.025

12. Brown MM, Brown GC, Sharma S, Landy J. Health care economic analyses and value-based medicine. Surv Ophthalmol. 2003;48 (2):204-223. doi:10.1016/S0039-6257(02)00457-5
13. Ross EL, Hutton DW, Stein JD, et al. Cost-effectiveness of aflibercept, bevacizumab, and ranibizumab for diabetic macular edema treatment: analysis from the diabetic retinopathy clinical research network comparative effectiveness trial. JAMA Ophthalmol. 2016;134(8):888-896. doi:10.1001/jamaophthalmol.2016.1669

14. NHS. National Institute for Health and Clinical Excellence. Guide to the methods of technology appraisal. Section 5.6: discounting. London. NICE; 2013. Available from: https://www.nice.org.uk/process/pmg9/ chapter/the-reference-case\#discounting. Accessed August 19, 2020.

15. Stein JD, Newman-Casey PA, Kim DD, Nwanyanwu KH, Johnson MW, Hutton DW. Cost-effectiveness of various interventions for newly diagnosed diabetic macular edema. Ophthalmology. 2013;120(9):1835-1842. doi:10.1016/j.ophtha.2013.02.002

16. Permsuwan U, Thavorn K, Dilokthornsakul P, Saokaew S, Chaiyakunapruk N. Cost-effectiveness of insulin detemir versus insulin glargine for Thai type 2 diabetes from a payer's perspective. $J$ Med Econ. 2017;20(9):991-999. doi:10.1080/13696998.2017.1347792

17. Li A, Rieveschl NB, Conti FF, et al. Long-term assessment of macular atrophy in patients with age-related macular degeneration receiving anti-vascular endothelial growth factor. Ophthalmol Retina. 2018;2(6):550-557. doi:10.1016/j.oret.2017.10.010

18. Virgili G, Parravano M, Evans JR, Gordon I, Lucenteforte E. Antivascular endothelial growth factor for diabetic macular oedema: a network meta-analysis. Cochrane Database Syst Rev. 2018;10: CD007419.

19. Ferris FL 3rd, Maguire MG, Glassman AR, Ying GS, Martin DF. Evaluating effects of switching anti-vascular endothelial growth factor drugs for age-related macular degeneration and diabetic macular edema. JAMA Ophthalmol. 2017;135(2):145-149. doi:10.1001/ jamaophthalmol.2016.4820

20. Holekamp N, Duff SB, Rajput Y, Garmo V. Cost-effectiveness of ranibizumab and aflibercept to treat diabetic macular edema from a US perspective: analysis of 2-year Protocol T data. J Med Econ. 2020;23(3):287-296. doi:10.1080/13696998.2019.1666855

21. Glassman AR, Wells JA 3rd, et al. Five-year outcomes after initial aflibercept, bevacizumab, or ranibizumab treatment for diabetic macular edema (Protocol T Extension Study). (published online ahead of print March 29, 2020). Ophthalmology. 2020;127(9):12011210. doi:10.1016/j.ophtha.2020.03.021.

22. Boyer DS, Nguyen QD, Brown DM, Basu K, Ehrlich JS. RIDE and RISE Research Group. Outcomes with as-needed ranibizumab after initial monthly therapy: long-term outcomes of the phase III RIDE and RISE trials. Ophthalmology. 2015;122(12):2504-2513.e1. doi:10.1016/j.ophtha.2015.08.006

23. Pieramici DJ, Wang PW, Ding B, Gune S. Visual and anatomic outcomes in patients with diabetic macular edema with limited initial anatomic response to ranibizumab in RIDE and RISE. Ophthalmology. 2016;123(6):1345-1350. doi:10.1016/j.ophtha.2016.02.007

24. Pieramici D, Singh RP, Gibson A, et al. Outcomes of diabetic macular edema eyes with limited early response in the VISTA and VIVID studies. Ophthalmol Retina. 2018;2(6):558-566. doi:10.1016/j. oret.2017.10.014

25. Bressler SB, Ayala AR, Bressler NM, et al. Persistent macular thickening after ranibizumab treatment for diabetic macular edema with vision impairment. JAMA Ophthalmol. 2016;134(3):278-285. doi:10.1001/jamaophthalmol.2015.5346

26. Fauser S, Muether PS. Clinical correlation to differences in ranibizu$\mathrm{mab}$ and aflibercept vascular endothelial growth factor suppression times. Br J Ophthalmol. 2016;100(11):1494-1498. doi:10.1136/ bjophthalmol-2015-308264

27. Kaiser PK. Prospective evaluation of visual acuity assessment: a comparison of snellen versus ETDRS charts in clinical practice (An AOS Thesis). Trans Am Ophthalmol Soc. 2009;107:311-324. 


\section{Publish your work in this journal}

Clinical Ophthalmology is an international, peer-reviewed journal covering all subspecialties within ophthalmology. Key topics include: Optometry; Visual science; Pharmacology and drug therapy in eye diseases; Basic Sciences; Primary and Secondary eye care; Patient Safety and Quality of Care Improvements. This journal is indexed on PubMed

Central and CAS, and is the official journal of The Society of Clinical Ophthalmology (SCO). The manuscript management system is completely online and includes a very quick and fair peer-review system, which is all easy to use. Visit http://www.dovepress.com/ testimonials.php to read real quotes from published authors. 